\title{
Criminologie
}

\section{La prévention des problèmes de comportement à l’adolescence : le Adolescent Transition Program}

\section{François Poulin, Thomas J. Dishion, Kate Kavanagh et Jeff Kiesner}

Volume 31, numéro 1, printemps 1998

La prévention de l'inadaptation sociale : approche développementale

URI : https://id.erudit.org/iderudit/017412ar

DOI : https://doi.org/10.7202/017412ar

Aller au sommaire du numéro

\section{Éditeur(s)}

Les Presses de l'Université de Montréal

ISSN

0316-0041 (imprimé)

1492-1367 (numérique)

Découvrir la revue

Citer cet article

Poulin, F., Dishion, T. J., Kavanagh, K. \& Kiesner, J. (1998). La prévention des problèmes de comportement à l'adolescence : le Adolescent Transition Program. Criminologie, 31(1), 67-85. https://doi.org/10.7202/017412ar
Résumé de l'article

The Adolescent Transition Program (ATP) is a multi-component preventive intervention designed to reduce escalation in problem behaviors among high-risk young adolescents. A previous evaluation of this program showed that aggregating high-risk youths for intervention purposes led to an escalation in delinquency and smoking. The results of this study also suggested that an intervention targeting parenting practices was more beneficial. These findings led to the development of a new intervention program aimed at the modification of parenting practices. This school-based program proposes a multiple gating approach to parent intervention with each level of intervention building on the previous one to reduce the overall prevalence of risk. A pilot study designed to evaluate the implementation of this intervention program suggested that schools seem to be an appropriate setting for reaching parents of high-risk adolescents and delivering intervention services.
Tous droits réservés @ Les Presses de l'Université de Montréal, 1998
Ce document est protégé par la loi sur le droit d'auteur. L’utilisation des services d'Érudit (y compris la reproduction) est assujettie à sa politique d'utilisation que vous pouvez consulter en ligne.

https://apropos.erudit.org/fr/usagers/politique-dutilisation/ 


\section{LA PRÉVENTION DES PROBLÈMES DE COMPORTEMENT À L'ADOLESCENCE : LE ADOLESCENT TRANSITION PROGRAM ${ }^{1}$}

François Poulin, Thomas J. Dishion, Kate Kavanagh et Jeff Kiesner ${ }^{2}$

The Adolescent Transition Program (ATP) is a multi-component preventive intervention designed to reduce escalation in problem behaviors among high-risk young adolescents. A previous evaluation of this program showed that aggregating high-risk youths for intervention purposes led to an escalation in delinquency and smoking. The results of this study also suggested that an intervention targeting parenting practices was more beneficial. These findings led to the development of a new intervention program aimed at the modification of parenting practices. This school-based program proposes a multiple gating approach to parent intervention with each level of intervention building on the previous one to reduce the overall prevalence of risk. A pilot study designed to evaluate the implementation of this intervention program suggested that schools seem to be an appropriate setting for reaching parents of high-risk adolescents and delivering intervention services.

Des études longitudinales révèlent que plusieurs des problèmes rencontrés à l'adolescence sont associés à une trajectoire de développement caractérisée par des comportements antisociaux. En effet, les comportements antisociaux constituent un important prédicteur, notamment, de la délinquance (Loeber et Dishion, 1983), de la consommation de drogues (Smith et Fogg, 1979) et des relations sexuelles précoces (Capaldi, Crosby et Stoolmiller, 1996). Tous ces problèmes de comportement tendent d'ailleurs à être corrélés entre eux à l'adolescence (Hawkins, Catalano et Miller, 1992). Une intervention ciblant les comportements antisociaux constitue donc une stratégie

1. Ce projet a été financé par une subvention du National Institute on Drug Abuse et du National Institute of Health (DA-07031) accordée au deuxième auteur, par une subvention du National Institute of Mental Health (MH-37940) accordée à John Reid, Ph.D., et par une subvention du National Institute of Mental Health (MH-46690) accordée à Gerald R. Patterson, Ph.D.

2. Oregon Social Leaming Center. Les demandes de tirés-à-part ou d'information peuvent être adressées à François Poulin, University of Oregon, Oregon Social Learning Center, 207 E. Fifth Ave. Suite 202, Eugene, OR 97401, U.S.A. Adresse électronique : francois@tigger.oslc.org 
prometteuse pour prévenir une augmentation de ces problèmes à l'adolescence.

Un consensus existe de plus en plus dans la littérature quant à l'importance d'adopter une perspective de développement dans la mise sur pied de stratégies d'intervention visant la prévention des comportements antisociaux (Coie, 1996 ; Reid et Eddy, sous presse ; Tolan, Guerra et Kendall, 1995). Plusieurs décennies de recherche ont mené à l'identification de caractéristiques individuelles, de processus et de conditions sociales qui sont associés aux comportements antisociaux à certaines périodes spécifiques du développement. Par exemple, des pratiques parentales sévères et coercitives caractérisées par une utilisation inefficace de la discipline contribuent à renforcer chez l'enfant l'utilisation de comportements antisociaux et coercitifs (Patterson, Reid et Dishion, 1992). Une fois atteint l'âge scolaire, l'école et le groupe de pairs constituent de nouveaux contextes susceptibles de favoriser le maintien des comportements antisociaux. En généralisant son style interactionnel coercitif, l'enfant se retrouve rapidement confronté au rejet par ses pairs et à des échecs scolaires (Coie, Belding et Underwood, 1988 ; Dishion, 1990). Ces expériences négatives amènent l'enfant à rechercher la compagnie de pairs antisociaux (Dishion, Patterson, Stoolmiller et Skinner, 1991). À l'adolescence, la fréquentation de pairs déviants constitue un facteur de risque majeur car ces derniers contribuent à renforcer l'adolescent dans son utilisation de comportements antisociaux et lui permettent d'apprendre de nouvelles formes de comportements déviants (Elliot, Huizinga et Ageton, 1985 ; Patterson, 1993). Par ailleurs, les parents continuent de jouer un rôle critique tout au long du développement. Par exemple, la qualité de leur supervision pendant l'adolescence peut influencer directement la réussite scolaire de l'adolescent (Patterson et al., 1992), sa fréquentation de pairs déviants (Dishion, Capaldi, Spracklen et Li, 1995 ; Patterson et Dishion, 1985), de même que sa consommation de drogues (Baumrind, 1985 ; Dishion et Loeber, 1985). Enfin, la qualité de la supervision exercée par les parents de même que l'accessibilité à des pairs déviants sont fortement influcncées par la composition de la communauté à laquelle appartiennent les familles (Patterson et al., 1992).

Les recensions des écrits portant sur la prévention des problèmes de comportement montrent que l'intervention la plus largement utilisée est la formation aux habiletés parentales (Kazdin, 1987 ; Patterson, Dishion et Chamberlain, 1993). L'efficacité de cette stratégie d'intervention a été démontrée notamment auprès des jeunes enfants présentant des troubles de comportement (Webster-Stratton, 1989) et des délinquants juvéniles (Bank, Marlowe, Reid, Patterson et Weinrott, 1991). De plus, les interventions centrées sur la famille qui visent à soutenir l'utilisation de pratiques parentales 
actives et constructives sont également efficaces pour réduire la consommation de drogues chez les adolescents à risque ëlevé (Bry, 1988 ; Szapocznik et Kurtines, 1989).

Par ailleurs, les interventions orientées directement vers les enfants ou les adolescents constituent également une stratégie répandue pour prévenir les problèmes de comportement. Ces interventions sont dans plusieurs cas guidées par des travaux de recherche mettant en évidence certains déficits observés chez les enfants et les adolescents antisociaux, sur le plan sociocognitif notamment, quant à leurs habiletés à résoudre des problèmes (Dodge, 1993). Des interventions visant à corriger ces déficits se sont avérées efficaces pour prévenir une augmentation des problèmes de comportement à l'adolescence (Guerra et Slaby, 1990 ; Lochman, 1992)

Bien qu'il soit souvent assumé qu'il est nécessaire d'intervenir tôt dans le développement de l'individu afin d'obtenir un effet préventif, le début de l'adolescence constitue une période stratégique pour une intervention préventive. En effet, il s'agit de la période pendant laquelle les problèmes de comportement sont les plus nombreux (Greenberg, 1991; Hirshi et Gottfredson, 1983). De plus, les jeunes qui présentent des problèmes de délinquance à cet âge sont à risque pour des problèmes plus sévères au début de l'âge adulte (Stattin et Magnusson, 1991). Enfin, la consommation de drogues au début de l'adolescence constitue un important prédicteur de problèmes de consommation plus sévères à l'âge adulte (Robins et Przybeck, 1985).

Cet article a comme objectif de présenter un programme d'intervention, le Adolescent Transition Program (ATP), conçu pour prévenir l'apparition deș problèmes de comportement chez les adolescents à haut risque. Il s'agit d'un programme en évolution offrant une variété de stratégies d'intervention. La première partie de l'article est consacrée à un rappel des travaux qui ont porté sur l'évaluation de l'efficacité de la première version du ATP. Cette première version comprenait deux composantes : une ciblant les pratiques parentales et une orientée directement vers les adolescents. La seconde partie de cet article est consacrée à la présentation d'une nouvelle version du ATP. Cette nouvelle version cible exclusivement les pratiques parentales associées aux comportements antisociaux de l'adolescent et intègre des interventions allant d'un niveau universel à un niveau individualisé. Son contenu, de même que les résultats d'une pré-expérimentation, sont présentés.

\section{ÉVALUATION DE L'EFFICACITÉ DE LA PREMIÈRE VERSION DU ADOLESCENT TRANSITION PROGRAM}

La première version du ATP a été mise sur pied selon une perspective sociale interactionelle du développement des comportements antisociaux 
(Patterson et al., 1992). La première composante de ce programme est une intervention parentale ciblant quatre habiletés de gestion familiale clefs : la supervision, l'utilisation de renforcements positifs, l'établissement de limites et la résolution de problèmes. La seconde composante est orientée directement vers les adolescents et vise à favoriser le développement de l'autorégulation et l'acquisition de compétences interpersonnelles utiles dans le milieu familial et dans le groupe de pairs. L'intervention parentale et l'intervention auprès des adolescents sont offertes en groupe, les groupes de parents et les groupes d'adolescents se rencontrant séparément pour une série de 12 séances hebdomadaires dirigées par des thérapeutes. Le contenu détaillé de ces séances est décrit par Dishion et Kavanagh (sous presse). Le choix et le développement de ces deux stratégies d'intervention ont été fait en se basant sur l'évaluation de l'efficacité de divers types d'intervention pour jeunes à haut risque (Kazdin, 1987 ; Patterson et al., 1993).

L'efficacité des composantes de cette première version du ATP a été évaluée auprès d'un échantillon de jeunes à haut risque (Dishion et Andrews, 1995). Les familles de 119 jeunes (âge moyen de 12 ans) ont été réparties aléatoirement dans quatre conditions différentes : (a) intervention auprès des parents seulement, (b) intervention auprès des adolescents seulement, (c) intervention auprès des parents et intervention auprès des adolescents combinées et (d) matériel auto-administré (vidéos et lettres d'information). De plus, 38 familles n'ayant reçu aucune intervention ont été suivies à titre de groupe contrôle quasi expérimental.

Des résultats à la fois encourageants et alarmants ressortent de cette étude. L'intervention parentale et l'intervention auprès des adolescents ont toutes deux eu un impact positif sur le fonctionnement de la famille. En effet, une diminution des interactions coercitives entre les parents et l'enfant de même qu'une diminution des conflits dans la famille ont été observées à la fin du traitement auprès des familles ayant reçu l'une ou l'autre de ces interventions (ou les deux) comparativement aux familles du groupe contrôle. De plus, l'intervention parentaie s'est avérée la plus efficace pour réduire les problèmes de comportement à l'école. Cependant, cet effet ne s'est pas maintenu au suivi d'un an.

Par ailleurs, des effets iatrogènes évidemment non planifiés et malheureux ont résulté de l'intervention impliquant la formation de groupes d'adolescents à risque. Un effet iatrogène est observé lorsqu'une intervention produit involontairement un effet négatif sur les participants. Ainsi, les adolescents qui ont participé à ces groupes rapportent consommer significativement plus de cigarettes à la fin de l'intervention et au suivi d'un an comparativement aux adolescents du groupe contrôle. De plus, un an après la fin de l'intervention, ces mêmes adolescents présentaient davantage de 
problèmes de comportement en milieu scolaire, tel que rapporté par l'enseignant, comparativement à ceux du groupe contrôle. Les effets iatrogènes résultant de la formation de ces groupes ont également été observés auprès des adolescents dont les parents participaient simultanément à l'intervention parentale.

Poulin et Dishion (1998) ont técemment examiné la durabilité de ces effets négatifs. Les sujets ayant pris part à l'étude ont été suivis annuellement sur une période de trois ans après la fin de l'intervention (taux de rétention de $85 \%$ ). La figure 1 illustre la fréquence de consommation de cigarettes des adolescents (transformation logarithmique) en fonction de leur participation à l'intervention de groupe. L'examen de cette figure révèle que l'effet iatrogène observé pour la consommation de cigarettes est toujours présent trois ans après la fin de l'intervention. De plus, les enseignants rapportent que les adolescents qui ont participé à l'intervention de groupe

Figure 1

Consommation de cigarettes des adolescents sur une période de trois ans suivant l'intervention ; $\mathrm{C} \approx$ contrôle ; $\mathrm{I}=$ Intervention auprès des adolescents

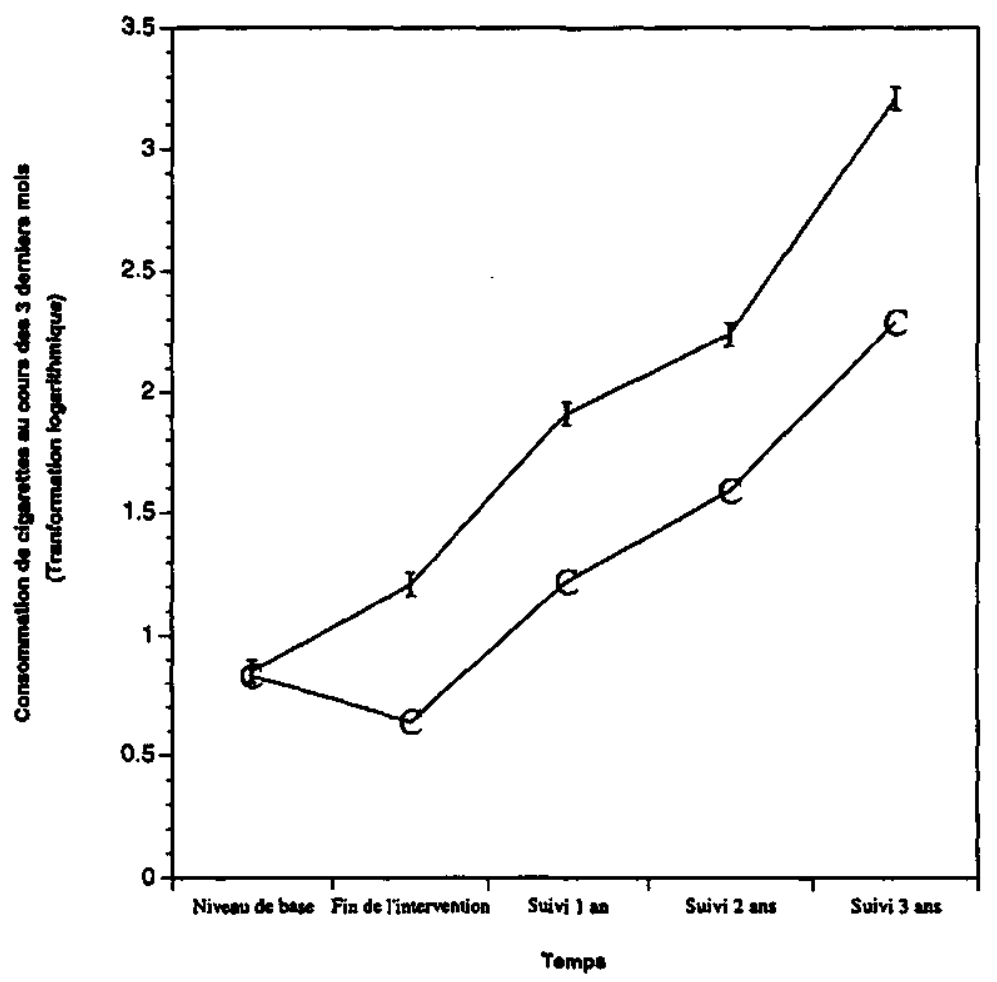


manifestent davantage de comportements délinquants trois ans plus tard comparativement à ceux du groupe contrôle (voir figure 2). En somme, l'effet négatif résultant de la formation d'un groupe d'adolescents à risque se maintient trois ans après la fin de l'intervention et est corroboré par deux sources d'information indépendantes, c'est-à-dire l'adolescent lui-même et les enseignants.

\section{Figure 2}

Comportements délinquants des adolescents sur une période de trois ans suivant l'intervention ; $\mathrm{C}=$ contrôle $\mathbf{I}$ = Intervention auprès des adolescents.

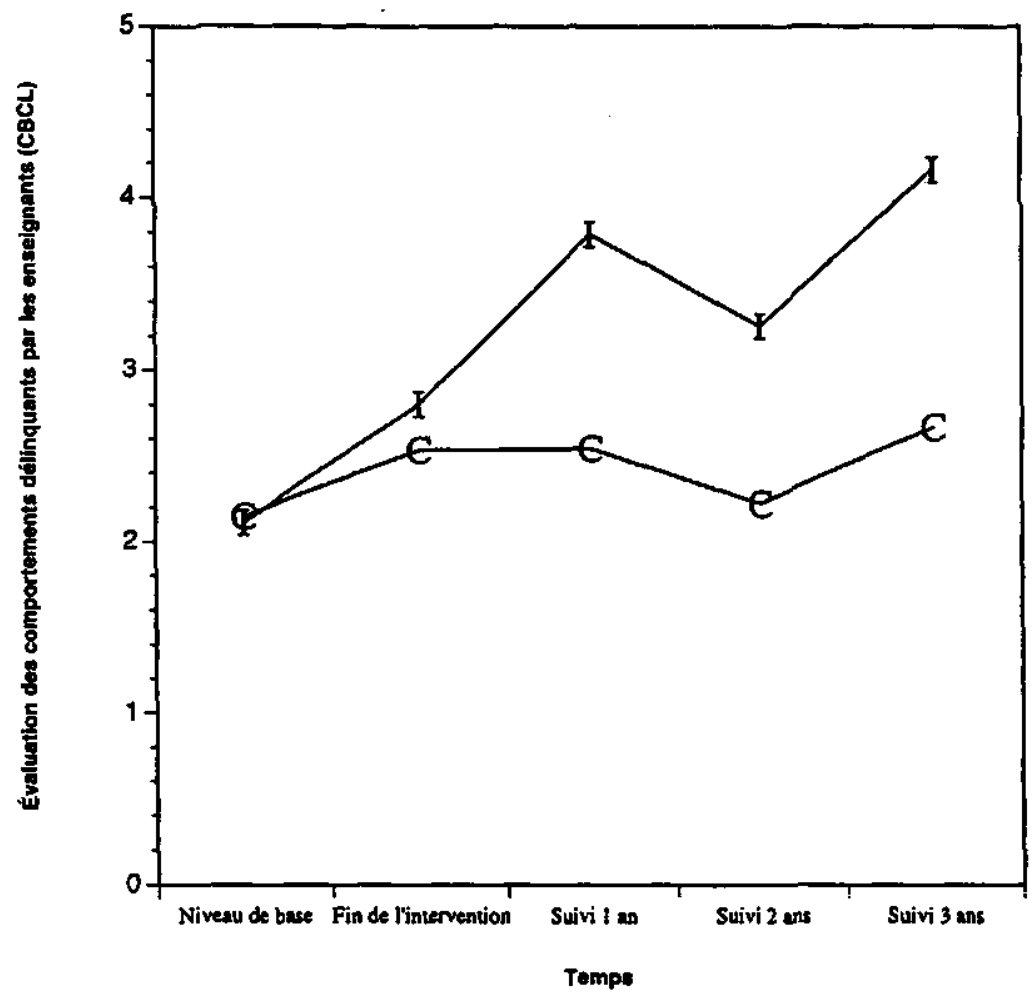

Ces résultats suggèrent que le regroupement d'adolescents à risque pour des fins d'intervention constitue une stratégie potentiellement dommageable. Il est à noter qu'il ne s'agit pas d'un cas isolé. La Cambridge-Somerville Youth Study (CSYS) qui avait comme objectif de prévenir la délinquance chez les jeunes à risque est un exemple classique d'effet iatrogène (McCord, 1978). Les jeunes qui ont pris part à cette intervention dans les années quarante présentaient plus de problèmes d'ajustement 30 ans plus tard que ceux du groupe contrôle. Dans une récente série d'analyses, McCord (1997) 
rapporte que la moitié des jeunes de la CSYS ont participé à des camps d'été pendant l'intervention. Or ces derniers présentent davantage de problèmes 30 ans plus tard comparativement aux jeunes qui n'ont pas pris part aux camps d'été. Il est donc possible que l'effet iatrogène observé dans la CSYS soit attribuable au regroupement de ces jeunes à risque dans des camps d'été.

Les résultats de ces deux études illustrent clairement les conséquences dommageables qui peuvent résulter du regroupement de jeunes à risque dans un but préventif. Une vaste littérature met en évidence le rôle des pairs déviants dans le développement des problèmes de délinquance et de consommation de drogue à l'adolescence (Elliot et al., 1985 ; Patterson, 1993). Il est possible que la formation de ces groupes à des fins d'intervention constitue de nouveaux contextes offrant aux adolescents à risque élevé des occasions d'établir des liens d'amitié entre eux. Une codification des enregistrements vidéoscopiques de chacune de ces séances est actuellement en cours pour mieux examiner les mécanismes associés à cet effet iatrogène. Le processus d'entraînement à la déviance identifié par Dishion, Spracklen, Andrews et Patterson (1996) pourrait opérer dans ces groupes.

Enfin, ces effets iatrogènes ont des implications importantes puisque le regroupement de jeunes à risque est une procédure très répandue en milieu scolaire, en institution et dans le cadre de diverses stratégies d'intervention. Il est essentiel que davantage de travaux soient menés afin de déterminer dans quelles circonstances la formation de ces groupes serait contre-indiquée. Par exemple, certaines études suggèrent que la formation de groupes composés d'enfants à risque et d'enfants pro-sociaux serait beaucoup plus bénéfique (Feldman, 1992 ; Tremblay, Pagani-Kurtz, Masse, Vitaro et Pihl, 1995).

Une des conséquences des résultats de cette évaluation empirique de la première version du ATP fut l'élimination de l'intervention de groupe auprès des adolescents. Par ailleurs, les résultats observés pour l'intervention parentale ont encouragé les auteurs à se concentrer sur cette stratégie d'intervention. D'autres travaux empiriques récents appuient également l'efficacité du volet parental du ATP (Irvine, Biglan, Metzler, Ary et Smolkowski, 1997). Par conséquent, la nouvelle version du ATP cible principalement les pratiques parentales associées au développement des problèmes de comportement chez les adolescents. Il s'agit d'un programme d'intervention à niveaux multiples qui intègre des interventions allant d'un niveau universel à un niveau individualisé.

\section{DESCRIPTION DU PROGRAMME D'INTERVENTION PARENTALE À NIVEAUX MULTIPLES IMPLANTÉ EN MILIEU SCOLAIRE}

Cette nouvelle version du ATP fut mise sur pied en adoptant une approche écologique des problèmes de comportement (Biglan, 1995 ; Kellam, 
1990). Un des principes de l'approche écologique soutient qu'il est important de travailler dans les milieux appropriés, notamment l'école. En servant de lieu de rencontre pour les groupes de pairs déviants et de terrains d'entraînement à leur déviance, le milieu scolaire exerce une influence importante sur les problèmes de comportement des adolescents (Dishion, Patterson et Griesler, 1994 ; Kellam, 1990 ; Rutter, 1985). De plus, le lien de communication que les parents entretiennent avec l'école a un impact sur la qualité de leur supervision, l'établissement de limites, de même que le soutien qu'ils offrent aux activités scolaires de leur enfant (Gottfredson, Gottfredson et Hybl, 1993 ; Reid, 1993). Par exemple, le simple fait d'acheminer davantage d'information aux parents sur les devoirs de l'enfant, de même que sur sa présence et son comportement en classe, contribue à améliorer la qualité de leur supervision de ses activités (Blechman, Taylor et Schrader, 1981; Heller et Fantuzzo, 1993). Il apparaît donc important de considérer les écoles comme site d'implantation de programmes d'intervention préventive (Trickett et Berman, 1989).

Ce programme comprend trois niveaux d'intervention préventive : 1) l'intervention universelle, qui cible toutes les familles d'une école désignée ; 2) l'intervention sélectionnée, qui cible les familles identifiées comme étant à risque, et 3) l'intervention individualisée, qui implique un soutien au changement plus intensif pour les familles présentant une problématique spécifique. Chaque niveau d'intervention s'ajoute au précédent de façon additive réduisant ainsi le niveau de risque. Le modèle est illustré à la figure 3 .

Figure 3

Une approche à niveaux multiples pour la prévention des problèmes de comportements chez les adolescents

\begin{tabular}{|c|c|c|}
\hline Nhvew 1 : intervention teniversele & Niveau 2: Intervention stlectionnd* & $\begin{array}{l}\text { Nhonu 3: intenvencion } \\
\text { nowidualiste }\end{array}$ \\
\hline $\begin{array}{l}\text { Objoctifs } \\
\text { Engugament } \\
\text { Auto-tivaliations } \\
\text { Supervision }\end{array}$ & $\begin{array}{l}\text { Obectits } \\
\text { Evaluation du riveas de risque } \\
\text { Engagement } \\
\text { Habllotes porentales }\end{array}$ & $\begin{array}{l}\text { Objectifs } \\
\text { Gestion du stress ot das crises } \\
\text { Trilitertent } \\
\text { Engegament }\end{array}$ \\
\hline $\begin{array}{l}\text { Stratiogies } \\
\text { Video sur les factoure de risque } \\
\text { Rencontre d'orientation } \\
\text { lione teltephanique }\end{array}$ & $\begin{array}{l}\text { Stratboles } \\
\text { Exomien de lo familite } \\
\text { Soution la mothation } \\
\text { Composantes du ATP } \\
\text { Info. tellephonkque hebdo. }\end{array}$ & 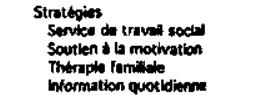 \\
\hline
\end{tabular}

L'objectif principal de l'intervention universelle est de favoriser la collaboration entre le personnel de l'école et les parents en mettant sur pied un centre de ressources pour les parents à l'intérieur de l'école. L'intervention sélectionnée s'adresse aux familles dont l'adolescent a été identifié par les enseignants comme étant à risque pour des problèmes de comportement futurs. Les parents participent à une entrevue dont l'objectif est de susciter la 
motivation au changement. Enfin, une petite proportion des parents vont avoir recours à un soutien professionnel direct afin de les aider à effectuer les changements identifiés lors de l'entrevue avec le consultant familial (niveau individualisé). En somme, chaque niveau d'intervention constitue une porte d'entrée pour le niveau suivant. Les modalités d'intervention spécifiques à chacun de ces niveaux sont décrites en détail dans les paragraphes qui suivent.

\section{Centre de ressources pour les parents (niveau 1)}

Un consultant familial est en poste à temps plein au centre de ressources pour les parents. Les activités offertes par ce centre sont conçues pour rejoindre les parents de tous les enfants de l'école. Durant la première semaine de la rentrée des classes, les parents participent à une soirée d'introduction au programme et sont par la suite invités à visionner une vidéocassette portant sur le rôle de parent lorsque l'enfant atteint l'âge de l'adolescence. Cet outil vise à initier chez eux un processus d'auto-évaluation permettant l'identification de leurs propres forces et faiblesses dans leurs interactions avec leur enfant. La vidéocassette présente des exemples de comportement à risque chez les adolescents et met l'accent sur l'utilisation de méthodes de gestion familiale efficaces (renforcement positif, surveillance, établissement de limites et habiletés relationnelles) permettant de composer avec ces problèmes. Suite au visionnement de cette vidéocassette, les familles (parents et enfants) sont invitées à compléter une série d'exercices élaborée par les consultants du centre de ressources pour les parents et les enseignant(e)s. Ces exercices visent à encourager et soutenir l'engagement des parents dans la vie de leur enfant, la communication parent-enfant et une gestion familiale saine.

Durant le reste de l'année scolaire, le centre de ressources pour les parents sert également de lien de communication entre l'école et les parents en acheminant à ces derniers des informations hebdomadaires sur les devoirs et les problèmes qui peuvent se présenter à l'école de même que sur les ressources disponibles. Enfin, ce centre constitue une ressource pour les membres du personnel de l'école qui sont soucieux de développer une relation de collaboration positive avec les parents.

\section{Entrevue motivationnelle (niveau 2)}

L'entrevue motivationnelle est une démarche en profondeur visant à aider les parents à évaluer avec précision le statut de risque de leur enfant et à soutenir leur intérêt et leur motivation à apporter les changements nécessaires. Elle comprend deux rencontres de deux heures chacune qui se déroulent au centre de ressources pour les parents. Le but de la première rencontre est d'évaluer divers aspects de la vie de l'enfant et de sa famille. Des 
informations sont alors recueillies sur les problèmes de comportement de l'enfant, les processus d'interaction et de communication parent-enfant, la surveillance parentale et le réseau d'amis de l'enfant. Les instruments de mesure utilisés pour procéder à cette évaluation sont présentés au tableau 1 . Lors de la seconde rencontre, les résultats sont présentés aux parents sous forme de comparaisons normatives afin de situer le niveau de risque de l'enfant et de la famille. Cette séance est structurée à partir du modèle FRAMES (Miller et Rollnick, 1991). Elle représente aussi une occasion de discuter avec les parents des dispositions qu'ils peuvent prendre pour améliorer la vie de leur famille et le bien-être de leur enfant.

\section{Tableau 1}

Construits mesurés dans le cadre de l'entrevue motivationnelle et instruments de mesure utilisés

Caractéristiques de l'adolescent

$\checkmark$ Parent Child Behavior Checklist (Achenbach, 1991)

$\checkmark$ Teacher Child Behavior Checklist (Achenbach, 1991)

$\checkmark$ Youth Self Report (Achenbach, 1991)

$\checkmark$ Child Depression Inventory (Kovacs, 1985)

$\checkmark$ Résultats scolaires

Relations de l'adolescent avec ses pairs

$\checkmark$ Parent Peers and Social Skills*

$\checkmark$ Peer involvement and Social Skills Questionnaire (enseignant ; Walker et McConnell, 1988)

$\checkmark$ Child's Peer Ratings*

Environnement familial

$\checkmark$ Family Events Checklist*

$\checkmark$ Life Events (Holmes et Rahe, 1967)

$\checkmark$ Parent Mood/Depression Questionnaire (Radloff, 1977)

$\checkmark$ Family Assessment Task*

* Disponible au Oregon Social Learning Center, 207 East 5th Avenue, Suite 202, Eugene, OR 97401.

Plusieurs familles ont déjà des forces qui compensent pour les facteurs de risque qu'elles présentent. L'entrevue motivationnelle leur procurera essentiellement un soutien supplémentaire aux efforts déjà investis. Dans le cas des familles présentant plus de difficultés, des conseils pratiques et un ensemble de stratégies correspondant à leurs besoins leur seront proposés.

\section{Intervention familiale (niveau 3)}

Ce niveau d'intervention comprend diverses stratégies utilisées par les thérapeutes familiaux d'approche comportementale, structurelle et éclectique 
travaillant auprès d'adolescents à risque (Dishion et Patterson, 1992 ; Forehand et McMahon, 1981 ; Bry, McGreene, Schutte et Fishman, 1991 ; Henggeler, Melton et Smith, 1992 ; Szapocznik et Kurtines, 1989). Le Systematic Screening for Behavior Disorders (Walker et Severson, 1991) est utilisé pour identifier les familles ayant besoin d'une intervention plus intensive. Les objectifs de l'intervention familiale de même que le type de services fourni aux parents sont déterminés avec leur collaboration. Certains parents n'ont besoin que de brèves interventions pour améliorer leurs habiletés de communication alors que d'autres pourraient tirer avantage d'une thérapie comportementale familiale plus intensive. Bien que la stratégie optimale soit de faire participer tous les membres de la famille dans l'intervention, il est recommandé de travailler avec quiconque est prêt à participer (Szapocznik, Perez-Vidal, Brickman, Foote, Santisteban et Hervis, 1988).

La première étape de l'intervention familiale consiste à aider les parents à identifier clairement et objectivement ce qui les préoccupe. Pour ce faire, ils sont invités à noter certains comportements problématiques de l'enfant lorsqu'ils surviennent à la maison et à l'école. Par la suite, des stratégies visant à renforcer le comportement opposé au comportement cible (c.-à.-d., plus prosocial) sont élaborées en collaboration avec les parents. On enseigne également aux parents comment utiliser l'information quotidienne provenant de l'école pour encourager la réussite scolaire de leur enfant. Pour plusieurs parents, la seconde étape consiste à cesser de réagir avec colère et sévérité lorsque leur enfant se conduit d'une manière désagréable et d'être plus constants dans l'établissement de limites. Une fois que ces habiletés sont en voie d'être acquises, les parents peuvent se concentrer davantage sur la surveillance et la supervision des allers et venues de leur enfant, particulièrement la fréquentation de pairs déviants. Enfin, il est important d'améliorer les habiletés de communication puisqu'il s'agit là de la base d'une relation parent-enfant positive et qu'elles sont cruciales dans la résolution des conflits (Forgatch, 1989).

Certains parents, notamment dans le cas de familles monoparentales, choisissent de participer à des séances de groupe et ainsi de bénéficier du soutien provenant d'autres parents. Un programme a été développé pour ces groupes sur la base du modèle de thérapie familiale comportementale (Dishion et Kavanagh, sous presse).

Enfin, tout au long du programme les intervenants sont supervisés dans le cadre de séances hebdomadaires d'études de cas. Dans la mesure du possible, les rencontres avec les familles sont enregistrées (vidéo ou audio). L'examen de ce matériel lors des études de cas permet d'évaluer le niveau d'engagement des parents dans le processus d'intervention et la qualité de la relation de collaboration qu'ils établissent avec les intervenants. Ces séances 
visent à fournir un soutien aux consultants responsables du cas et à les aider à trouver et implanter des stratégies d'intervention efficaces. De plus, elles contribuent au développement d'une expertise et d'un esprit de soutien dans l'équipe clinique.

\section{IMPLANTATION DE L'INTERVENTION PARENTALE À NIVEAUX MULTIPLES EN MILIEU SCOLAIRE}

L'êvaluation de cette intervention a récemment été entreprise. Puisque ce programme a été conçu selon une approche écologique des problèmes de comportement et cible les écoles comme site d'intervention, une première étape consistait à évaluer la faisabilité d'une telle démarche en milieu scolaire. En effet, il est primordial de déterminer si l'école constitue un site adéquat pour rejoindre les parents des adolescents à risque et leur offrir des services d'intervention. Une pré-expérimentation a donc été menée à cette fin.

Deux écoles de premier cycle du secondaire et une école de second cycle ont pris part à cette pré-expérimentation. Ces écoles sont situées dans une communauté à haut risque du nord-ouest des États-Unis où le niveau socioéconomique des familles est en général faible. Quatre-vingt-dix pour cent des élèves qui fréquentent ces écoles sont d'origine caucasienne, les autres étant principalement d'origine amérindienne ou espagnole.

Le premier niveau de cette intervention, le niveau universel, a consisté à établír un centre de ressources pour les parents dans chacune de ces trois écoles. Les activités de ce centre étaient assurées par un consultant familial. De l'information décrivant les services offerts par ce centre a été acheminée aux parents par l'entremise de leur enfant. Les parents de 118 adolescents ont fréquenté un des centres établis dans les trois écoles sur une base volontaire et ont emprunté et visionné la vidéocassette d'information (une étude est actuellement en cours afin d'évaluer l'impact du visionnement de cette vidéocassette sur les pratiques parentales). Cinquante d'entre eux ont rencontrć le consultant, encore une fois sur une base volontaire, pour discuter de divers problèmes concernant leur enfant. La nature de ces problèmes de même que la proportion des parents qui les ont rapportés sont présentées au tableau 2. L'examen de ce tableau révèle que les préoccupations étaient principalement centrées autour des résultats scolaires et de la réalisation des devoirs. Le second type de préoccupations le plus courant concernait la qualité des relations parents-enfants à la maison. Les problèmes de comportement de l'enfant étaient également un thème fréquent. Les parents semblaient donc à l'aise d'aborder une grande variété de problèmes avec le consultant familial.

Parmi les familles qui ont rencontré un consultant, 16 ont participé au deuxième niveau d'intervention, c'est-à-dire à l'entrevue motivationnelle. 
Tableau 2

Problèmes caractêrisant les adolescents dont les parents ont consulté le centre de ressources pour la farnille

\begin{tabular}{lc}
\hline Type de problème & $\begin{array}{c}\text { Pourcentage de famille qui } \\
\text { ont identifí ce problème }\end{array}$ \\
\hline Résultats scolaires / devoirs & $71 \%$ \\
Conflit parent / enfant & $33 \%$ \\
Problèmes de comportement à l'école & $30 \%$ \\
Colère / violence & $29 \%$ \\
Perte d'un parent & $17 \%$ \\
Conflit avec ses pairs & $13 \%$ \\
Manque d'habiletés sociales / peu d'amis & $13 \%$ \\
Problèmes d'alimention / sommeil & $13 \%$ \\
Séparation / divorce & $13 \%$ \\
Consommation de cigarettes / alcool / drogue & $8 \%$ \\
Conflit avec ses frères / sceurs & $8 \%$ \\
\hline
\end{tabular}

Note. La somme de ces pourcentages exède 100 parce que certaines familles ont identifié plus d'un problème.

En se basant sur la sévérité des problèmes rapportés par les parents, les consultants ont identifié les familles qui étaient les plus susceptibles de bénéficier de cette intervention. Tel que décrit précédemment, la première séance était consacrée à l'évaluation de divers aspects de la vie de l'enfant et de sa famille. Les résultats étaient communiqués aux parents lors de la seconde rencontre et servaient de tremplin pour lancer la discussion sur les dispositions à prendre pour améliorer la vie de leur famille et le bien-être de leur enfant.

Bien que l'objectif principal de cette pré-expérimentation n'était pas d'évaluer de façon systématique dans quelle mesure cette entrevue s'avérait efficace pour susciter la motivation au changement chez les parents, certaines données s'avèrent tout de même encourageantes. Ainsi, pour un grand nombre de familles ces séances ont constitué une porte d'entrée vers le troisième niveau d'intervention qui implique un recours à des démarches plus approfondies. En effet, huit de ces familles ont consulté des ressources à l'extérieur de l'école comme il leur avait été suggéré lors de l'entrevue motivationnelle. De plus, quatre d'entre elles ont pris part à des ateliers portant sur la gestion familiale et le développement d'habiletés interpersonnelles offerts par le centre de ressources pour les parents.

Un sondage d'impact et un sondage d'utilisation ont été menés afin d'évaluer la réaction du milieu scolaire et des parents face à l'implantation de ce programme dans les écoles. Le personnel et les administrateurs des 
écoles ont indiqué que le centre de ressources pour les parents offrait plusieurs bénéfices à l'école et aux familles, notamment : (a) la possibilité de rencontrer les consultants du centre de ressources ; (b) l'amélioration de la qualité et de la justesse de l'information échangée entre les parents et les enseignant(e)s, et (c) l'augmentation de l'implication des parents dans les progrès scolaires de leur enfant. Par ailleurs, les parents qui ont fréquenté le centre de ressources ont rapporté que l'accès à ce centre en milieu scolaire leur avait permis de discuter de diverses préoccupations familiales. De plus, ces parents ont également rapporté être plus ouverts à recevoir de l'information concernant leur enfant en provenance de l'école.

En somme, les résultats de cette pré-expérimentation tendent à appuyer le choix de l'école comme site d'implantation pour rejoindre les parents des adolescents à risque et leur offrir des services d'intervention. Cependant, cette pré-expérimentation ne fournit aucune indication quant à l'efficacité de cette intervention pour la prévention des problèmes de comportement auprès des adolescents à risque.

Une des composantes de ce programme, l'entrevue motivationnelle, a récemment reçu un premier soutien empirique (Rao, 1997). Les familles de 20 garçons et de 20 filles (âge moyen de 12,77 ans ; $95 \%$ caucasien ; niveau de risque élevé) provenant d'une communauté du nord-ouest américain ont été réparties aléatoirement dans les deux conditions suivantes : a) examen de la famille; ou b) liste d'attente. Les familles de la condition expérimentale ont participé à une séance d'évaluation et à une séance de motivation. Une diminution significative des problèmes d'extériorisation perçus par le père et la mère a été observée chez les jeunes dont la famille a pris part à cette intervention comparativement à ceux du groupe contrôle. De plus, les parents ont rapporté utiliser davantage d'habiletés de gestion de la famille suite à la séance de rétroaction. Cependant, aucune différence significative n'a été observée entre les deux groupes sur les problèmes d'extériorisation évalués par l'enseignant ou auto-évalués par l'adolescent. Les résultats de cette étude suggère qu'il s'agit là d'une stratégie d'intervention prometteuse pour motiver les familles au changement. Cependant, il sera important de vérifier si les effets bénéfiques résultant d'une telle intervention au début de l'adolescence se maintiennent à long terme puisque les problèmes de comportement que l'on souhaite prévenir, notamment la délinquance et la consommation de drogues, se manifestent plus tard à l'adolescence.

\section{CONCLUSION}

L'objectif de cet article était de faire le point sur l'évolution d'un programme d'intervention, le Adolescent Transition Program, visant la 
prévention des problèmes de comportement à l'adolescence. Les travaux portant sur l'évaluation de l'efficacité de la première version de ce programme ont conduit à deux conclusions importantes. Premièrement, une intervention impliquant le regroupement de jeunes à risque s'avère une stratégie peu indiquée. Les effets iatrogènes résultant de cette intervention ont entraîné l'élimination de cette composante du menu des activités du ATP. Deuxièmement, les résultats de l'intervention ciblant les pratiques parentales suggèrent qu'il s'agit d'une stratégie plus efficace pour prévenir une augmentation des problèmes de comportement à l'adolescence. Bien que cette conclusion soit également appuyée par d'autres travaux (Bank et al., 1991 ; Henggeler et al., 1992 ; Szapocznik et Kurtines, 1989), cibler les pratiques parentales à l'adolescence demeure encore une stratégie peu exploitée. Une des difficultés associées à l'implantation d'une intervention parentale est la qquestion de l'engagement. Le modèle décrit dans la deuxième partie de cet article suggère qu'une telle intervention devrait être étroitement enchâssée dans le contexte scolaire afin d'atteindre les parents des jeunes à risque élevé et ainsi de maximiser son efficacité. Cette approche est d'ailleurs de plus en plus utilisée pour rejoindre les parents des jeunes en difficulté (p. ex., FAST-TRACK ; McMahon et Slough, 1996 ; LIFT ; Reid, 1996)

Par ailleurs, l'efficacité du centre de ressources pour les parents et de l'entrevue motivationnelle reste encore à démontrer. Les services offerts par le centre de ressources pour les parents visent à éduquer les parents sur les facteurs de risque, à mobiliser l'utilisation des ressources parentales et, si possible, à augmenter la surveillance générale exercée par les parents sur les progrès scolaires de leur enfant. Par contre, l'entrevue motivationnelle peut avoir des effets plus profonds et persistants semblables à ceux observés lorsque cette démarche est appliquée aux adultes présentant des problèmes d'alcoolisme (Miller et Rollenick, 1991). Le cas échéant, une motivation à changer de plus en plus élevée peut contribuer à enclencher un cycle de modification de comportements qui ne soit pas dépendant de contacts avec un thérapeute individuel ou un aidant. Plusieurs parents peuvent choisir avec succès de changer par eux-mêmes alors que d'autres pourront demander ou nécessiter une intervention familiale plus élaborée. L'étude de Rao (1997) suggère que l'entrevue motivationnelle constitue une stratégie d'intervention fort prometteuse.

Enfin, avant de tirer toute conclusion quant à son efficacité pour prévenir les problèmes de comportement auprès des adolescents à risque, ce programme d'intervention parentale à niveaux multiples implanté en milieu scolaire doit faire l'objet d'évaluations empiriques systématiques. Les résultats de ces travaux auront des implications importantes quant aux changements à apporter dans la façon dont les interventions sont implantées en milieu 
scolaire et quant à l'ajout de nouvelles stratégies d'intervention, efficaces et peu coûteuses, à l'ensemble des outils de prévention déjà existants.

\section{BIBLIOGRAPHIE}

ACHENBACH, T. M. (1991), Manual for the Child Behavior Checklist/4-18 and 1991 profile, Burlington, VT, University of Vermont, Department of Psychology.

BANK, L., MARLOWE, J. H., REID, J. B., PATTERSON, G. R. et WEINROTT, M. A. (1991), "Comparative evaluation of parent training for families of chronic delinquents ", Journal of Abnormal Child Psychology, vol. 19, p. 15-33.

BAUMRIND, D. (1985), " Familial antecedents of adolescence drug use : A developmental perspective ", in C.L. JONES et R.J. BATTJES (dir.), Etiology of Drug Abuse : Implications for prevention, (p. 13-44), Washington, DC, U.S. Government Printing Office.

BIGLAN, A. (1995), A Contextual Framework for Changing Cultural Practices, Reno, NV, Context Press.

BLECHMAN, E.A., TAYLOR, C.J. et SCHRADER, S.M. (1981), « Family problem solving versus home notes as early intervention with high-risk children ", Journal of Consulting and Clinical Psychology, vol. 49, p. 919-926.

BRY, B. (1988), « Family-based approaches to reducing adolescent substance use : Theories, techniques, and findings ", in RAHDERT, E. R. et GRABOWSKI, J. (dir.), Adolescent Drug Abuse : Analyses of treatment research, (p. 39-69), Rockville, MD, National Institute on Drug Abuse.

BRY, B. H., MCGREENE, D., SCHUTTE, C. et FISHMAN, C. A. (1991), Targeted Family Intervention Manual, Manuscrit non publié, Princeton, NJ, Rutgers State University.

CAPALDI, D.M., CROSBY, L. et STOOLMILLER, M. (1996), «Predicting the timing of first sexual intercourse for at-risk adolescent males ", Child Development, vol. 67, p. 344-359.

COIE, J.D. (1996), " Prevention of violence and antisocial behavior », in PETERS, R. D. et McMAHON, R. J. (dir..), Preventing Childhood Disorders, Substance Abuse, and Delinquency (p. 1-18), Thousand Oaks, CA, SAGE Publications.

COIE, J.D., BELDING, M. et UNDERWOOD, M. (1988), "Aggression and rejection in childhood ", in LAHEY, B. B. et KAZDIN, A. E. (dir.), Advances in Clinical Child Psychology, (p. 125-158), New York, Plenum Press.

DISHION, T.J. (1990), A developmentai model for peer relations ; Middle childhood correlates and one-year sequelae, Thèse de doctorat non publiée, Univerity of Oregon, Eugene.

DISHION, T. J. et ANDREWS, D. W. (1995), « Preventing escalation in problem behaviors with high-risk young adolescents : Immediate and 1-year outcomes", Journal of Consulting and Clinical Psychology, vol. 63, p. 538-548.

DISHION, T. J., CAPALDI, D., SPRACKLEN, K. M. et LI, F. (1995), « Peer ecology of male adolescent drug use ", Development and Psychopathology, vol. 7, p. 803-824.

DISHION, T. J. et KAVANAGH, K. (sous presse), Adolescent Transition Program : Assessment and intervention sourcebook, New York, Guilford Press.

DISHION, T.J. et LOEBER, R. (1985), « Adolescent marijuana and alcohol use : The role of parent and peers revisited ", American Journal of Drug and Alcohol Abuse, vol. 11, p. 11-25. 
DISHION, T. J. et PATTERSON, G. R. (1992), " Age effects in parent training outcome ", Behavior Therapy, vol. 23, p. 719-729.

DISHION, T. J., PATTERSON, G. R. et GRIESLER, P. C. (1994), « Peer adaptation in the development of antisocial behavior : A confluence model », in HUESMANN, L. R. (dir.), Aggressive Behavior : Current Perspectives, (p. 61-95), New York, Plenum Press.

DISHION, T. J., PATTERSON, G. R., STOOLMILLER, M. et SKINNER, M. (1991), « Family, school, and behavioral antecedents to early adolescent involvement with antisocial peers », Developmental Psychology, vol. 27, p. 172-180.

DISHION, T.J., SPRACKLEN, K.M., ANDREWS, D.W. et PATTERSON, G.R. (1996), "Deviancy training in male adolescent friendships ", Behavior Therapy, vol. 27, p. 373-390.

DODGE, K.A. (1993), "Social-cognitive mechanisims in the development of conduct disorder and depression ", Annual Review of Psychology, vol. 44, p. 559-584.

ELLIOTT, D. S., HUIZINGA, D. et AGETON, S. S. (1985), Explaining Delinquency and Drug Use, Beverly Hills, CA, Sage.

FELDMAN, R.A. (1992), “ The St. Louis Experiments : Effective treatment of antisocial youths in prosocial peer groups ", in McCORD, J. et TREMBLAY, R. E. (dir.), Preventing Antisocial Behavior, (p. 233-252), New York, Guilford Press.

FOREHAND, R. et MCMAHON, R. J. (1981), Helping the Noncompliant Child : A clinician's guide to parent training, New York, Guilford Press.

FORGATCH, M.S. (1989), "Patterns and outcome in family problem solving: The disrupting effect of negative emotion ", Journal of Marriage and Family, vol. 51, p. 115-124.

GOTTFREDSON, D. C., GOTTFREDSON, G. D. et HYBL, L. G. (1993), « Managing adolescent behavior: A multiyear, multischool study ", American Educational Research Journal, vol. 30, p. 179-215.

GREENBERG, D. (1991), « Modeling criminal careers ", Criminology, vol. 29, p. 17-46.

GUERRA, N.G. et SLABY, R.G. (1990), "Cognitive mediators of aggression in adolescent offenders : Inlervention ", Developmental Psychology, vol. 26, p. 269-277.

HAWKINS, J. D., CATALANO, R. F. et MILLER, J. Y. (1992), « Risk and protective factors for alcohol and other drug problems in adolescence and early adulthood: Implications for substance abuse prevention ", Psychological Bulletin, vol. 112, p. 64105 .

HELLER, L. R. et FANTUZZO, J. W. (1993), « Reciprocal peer tutoring and parent partnership : Does parent involvement make a difference? "School Psychology Review, vol. 22, p. 517-534.

HENGGELER, S. W., MELTON, G. B. et SMITH, L. A. (1992), «Family preservation using multisystemic treatment : An effective alternative to incarcerating serious juvenile offenders », Journal of Consulting and Clinical Psychology, vol. 60, p. 953-961.

HIRSCHI, T. et GOTTFREDSON, M. (1983), "Age and the explanation of crime", American Journal of Sociology, vol. 89, p. 552-584.

HOLMES, T.H. et RAHE, R.H. (1967), " The social readjustment rating scale », Journal of Psychosomatic Research, vol. 11, p. 213.

IRVINE, A.B., BIGLAN, A., METZLER, C.W., ARY, D.A. et SMOLKOWSKI, K. (1997), The Effectiveness of a Parenting Skills Program for Parents of Middle School Students in Small Communities, manuscrit non publié, Oregon Research Institute, Eugene, Oregon. 
KAZDIN, A.E. (1987), "Treatment of antisocial behavior in children : Current status and future directions ", Psychological Bulletin, vol. 102, p. 187-203.

KELLAM, S. G. (1990), Developmental epidemiological framework for family research on depression and aggression ", in PATTERSON, G. R. (dir.), Depression and Aggression in Family Interaction, (p. 11-48), Hillsdale, NJ, Erlbaum.

KovaCS, M. (1985), "The Children's Depression Inventory", Psychopharmacology Bulletin, vol. 21, p. 995-999.

LOCHMAN, J.E. (1992), « Cognitive-behavioral intervention with aggressive boys : Threeyear follow-up and preventive effects ", Journal of Counsulting and Clinical Psychology, vol. 60, p. 426-432.

LOEBER, R. et DISHION, T.J. (1983), « Early predictors of male delinquency : A review ", Psychological Bulletin, vol. 94, p. 68-99.

MCCORD, J. (1978), "A thirty-year follow-up of treatment effects", American Psychologist, vol. 33, p. 284-289.

MCCORD, J. (1997, juin), Some Unanticipated Consequences of Summer Camps, communication présentée à la rencontre bisannuelle de la Society for Research in Child Development, Washington, DC.

MCMAHON, R.J. et SLOUGH, N.M. (1996), « Family-based intervention in the Fast Track program ", in PETERS, R. D. et McMAHON, R. J. (dir.), Preventing Childhood Disorders, Substance Abuse, and Delinquency, (p. 90-110), Thousand Oaks, CAL, Sage.

MILLER, W. R. et ROLLNICK, S. (1991), Motivational Interviewing : Preparing people to change addictive behavior, New York, Guilford Press.

PATTERSON, G.R. (1993), "Orderly change in a stable world : The antisocial trait as a chimera ", Journal of Consulting and Clinical Psychology, vol. 61, p. 91 1-919.

PATTERSON, G. R. et DISHION, T. J. (1985), " Contributions of families and peers to delinquency ", Criminology, vol. 23, p. 63-79.

PATTERSON, G. R., DISHION, T. J. et CHAMBERLAIN, P. (1993), "Outcomes and methodological issues relating to treatment of antisocial children $m$, in GILES, T. R. (dir.), Handbook of Effective Psychotherapy, (p. 43-88), New York, Plenum.

PATTERSON, G. R., REID, J. B. et DISHION, T. J. (1992), Antisocial Boys, Eugene, OR, Castalia.

POULIN, F. et DISHION, T.J. (1998), Iatrogenic Effects among High Risk Adolescents Aggregated within Interventions : An analysis of the durability and process. Manuscrit en préparation.

RADLOFF, L.S. (1977), « The CED-D scale : A self-report depression scale for research in the general population ", Applied Psychology Measurement, vol. 1, p. 385-401.

RAO, S. (1997), An Evaluation of a Brief Motivational lnterview for Parents of High-Risk Children : The family check-up, thèse de doctorat non-publiêe, University of Oregon, Eugene, Oregon.

REID, J. B. (1993), “ Prevention of conduct disorder before and after school entry : Relating interventions to developmental findings », Development and Psychopathology, vol. 5, p. 243-262.

REID, J.B. (1996), Efficacy Trials in the Prevention of Conduct Disorder, communication présentée dans le cadre du Fifth National Institute of Mental Health National Conference on Prevention Research, Washinglon, D.C. 
REID, J.B. et EDDY, M. (sous presse), « The prevention of antisocial behavior : Some considerations in the search for effective interventions ", in D.M. STOFF, BREILING, J. et MASER, J. D. (dir.), The Handbook of Antisocial Behavior, New York, John Wiley and Sons.

ROBINS, L. N. et PRZYBECK, T. R. (1985), « Age of onset of drug use as a factor in drug and other disorders ", in JONES, C. L. et BATTJES, R. J. (dir.), Etiology of Drug Abuse : Implications for prevention, (p. 178-193), National Institute on Drug Abuse Research Monograph No 56, Washington, DC, Supt. of Docs., U.S. Govt. Print. Off.

RUTTER, M. (1985), “ Family and school influences on behavioral development », Journal of Child Psychology and Psychiatry, vol. 26, p. 349-368.

SMITH, G. M. et FOGG, C. P. (1979), « Psychological antecedents of teenage drug use », Research on Community and Mental Health, vol. 1, p. 87-102.

STATTIN, H. et MAGNUSSON, D. (1991), «Stability and change in criminal behavior up to age 30 », British Journal of Criminology, vol. 31, p. 327-346.

SZAPOCZNIK, J. et KURTINES, W.M. (1989), Breakthroughs in Family Therapy with Drug-Abusing and Problem Youth, New York, Springer.

SZAPOCZNIK, J., PEREZ-VIDAL, A., BRICKMAN, A. L., FOOTE, F. H., SANTISTEBAN, D. et HERVIS, O. (1988), « Engaging adolescent drug abusers and their families in treatment: A strategic structural systems approach », Journal of Consulting and Clinical Psychology, vol. 56, p. 552-557.

TOLAN, P.H., GUERRA, N.G. et KENDALL, P.C. (1995), « A developmental-ecological perspective on antisocial behavior in children and adolescents : Toward a unified risk and intervention framework ", Journal of Consulting and Clinical Psychology, vol. 63, p. 579-584.

TREMBLAY, R.E., PAGANI-KURTZ, L., MASSE, L.C., VITARO, F. et PIHL, R.O. (1995), " A bimodal preventive intervention for disnuptive kindergarten boys : Its impact through mid-adolescence ", Journal of Consulting and Clinical Psychology, vol. 63, p. 560-568.

TRICKETT, E. J. et BERMAN, D. (1989), « Taking ecology seriously : A community development approach to individually based preventive interventions in the schools ", in BOND, L. A. et COMPAS, B. E. (dir.), Primary Prevention and Promotion in the Schools, (p. 361-390), Newbury Park, CA, Sage.

WALKER, H. M. et SEVERSON, H. H. (1991), Systematic Screening for Behavior Disorders : Training manual, Longmont, $\mathrm{CO}$, Sopris West.

WALKER, H. et MCCONNELL, S. (1988), The Walker-McConnell Scale of Social Competence and School Adjusment, Austin, TX, Pro-Ed Publishers.

WEBSTER-STRATTON, C. (1989), "Systematic comparison of consummer satisfaction of three cost-effective parent training programs for conduct problem children ", Behavior Therapy, vol. 20, p. 103-116. 